\title{
COMMON PHYSICAL PROCESSES IN NATURAL AND TRIGGERED LIGHTNING IN WINTER STORMS IN JAPAN
}

\author{
Zen-Ichiro Kawasaki \\ Osaka University, Japan \\ Vladislav Mazur \\ NOAA/ERL/National Severe Storms Laboratory \\ Norman Oklahoma, USA
}

\begin{abstract}
Analysis of measurements of electric, magnetic, and radiation field variations produced by rocket-triggered discharges with trailing and grounded wire in winter storms in Japan revealed different physical processes than those found in rocket-triggered flashes in summer thunderstorms with negative fields overhead. It is shown that these discharges are negative leaders that consist of continuous current and a pulse process associated with streamer development. The negative leader is not followed by a process analogous to a return stroke in cloud-to-ground flashes. Analysis of data and application of the electrostatic model of lightning as a bidirectional and uncharged leader in an ambient electric field show the commonality of processes in rocket-initiated discharges and in positive cloud-to-ground flashes after the positive leader touches the ground.
\end{abstract}

\section{Introduction}

The rocket-triggering lightning technique used since 1970 s presents a unique opportunity to investigate the physics of lightning in a controlled environment. The processes taking place inside a cloud following development of a leader and between return strokes are still poorly understood. Despite the growing number of observations of positive CG flashes, we still far from understanding the difference in characteristics of positive and negative CG flashes. Studies of rocket-triggered flashes in winter storms with a positive charge overhead reveal somewhat different processes than those in rocket-triggered flashes in summer thunderstorms with a negative space charge overhead. In this paper we analyze electromagnetic waveforms of natural and rocket-triggered flashes in winter storms in Japan to find common physical processes in them. These observations and instruments used were briefly described by Kawasaki et al. [1991].

\section{Natural Positive CG Flashes}

In E-field records of positive CG flashes, the initiation process begins with a series of pulses and a slope (Fig. 1). The polarity of pulses is always the same as the slope direction: in observed cases of natural positive CG flashes, the pulse polarity is negative and positive leaders produce a negative E-field slope (Fig. 2). The same polarity of both pulses and a slope is consistent with the bidirectional leader concept [Kasemir, 1950; 1960] : because negative and positive leaders are parts of one system of conducting lightning channels, both the pulse current and continuous current flow in the same direction, although the positive leader advances toward the ground, whereas the negative leader propagates into the cloud and away from an observer on the ground.

The duration of individual negative stepped leader pulses, a few microseconds, and their rate, tens of pulses per milliseconds, in E-field records during the flash initiation [Kawasaki et al., 1991], correspond well to description of 
radiation field waveforms produced by lightning stepped leaders [Krider and Radda, 1975]. Evidence that the negative leader continues longer than that seen in the E-field record and as long as the duration of the positive leader (between its initiation and occurrence of return stroke) is found in records of UHF radiation (Fig. 3 ).

A return stroke follows a positive leader after the leader reaches the ground. Most return strokes have E-field changes that oscillate one or two harmonics before the process becomes aperiodic. The amplitude of the second harmonic is usually less than a one-half the amplitude of first (Fig. 4). An interval between peaks varies from several microseconds to a few tens of microseconds. Other than one or two well pronounced harmonics, there is a noticeable lack of high- frequency (higher than the oscillation mentioned earlier) variation in the return stroke waveform. The high-frequency variation in first return strokes of negative CG flashes is attributed [LeVine and Moneghini, 1983] to tortuosity and branching of the return stroke channel. The oscillating character of E-field changes immediately brings to mind an idea of reflected current waves in the return stroke channel [Kasemir, 1960; Takagi and Takeuti, 1983].

The E-field variation following the return stroke (Fig. 3) has a much steeper slope than that before the return stroke. This indicates a surge of continuous current in the "old" channel of the positive leader. Then the E-field record reaches its maximum negative value $(\mathrm{dQ} / \mathrm{dt}=\mathrm{I}=0)$, which means that the continuous current stops and thus the lightning channel is cut off from the ground. Records of UHF radiation (e.g., Fig. 3) reveal that high-rate radiation is observed both before and after the return stroke. In fact, radiation intensifies immediately following the return stroke, decreases slowly during the period of continuous current flow, and is still present after the lightning channel is cut off (e.g., Fig. 5). Knowing that UHF radiation with a pulse rate up to $100 \mathrm{~ms}^{-1}$ is associated with negative leader development, we conclude that there is a regeneration of the negative stepped leader after the return stroke. The continuous current during this stage of the flash is a part of the negative leader process. The duration of this continuous current starting from the moment of return stroke until the channel cutoff is several milliseconds to several tens of milliseconds.

\section{Discharges Initiated by Rockets with Trailing and Grounded Wire}

A11 but one of these discharges were initiated under the positive space charge overhead (positive E-field on the ground). The typical E-field record of a rocket-initiated discharge in winter storms has a bell-shape waveform, followed by a slow recovery slope (Fig. 6). The initiation process is seen as a combination of a continuous slow field change with gradually increasing slope and a pulse series (in records of the UHF radiation and magnetic current density variation, Fig 6). Radiation pulses are similar to those associated with streamers (steps) of negative leader.

The slow E-field change is indicative of the continuous current of the negative leader. The duration of the downward slope of the E-field change to its maximum corresponds to the duration of continuous current flow from the beginning of the leader to a current cutoff point. UHF radiation records (Fig. 6) show that (1) the period of intensifying radiation (high pulse rate and amplitude) corresponds 
to the period of the increasing E-field (downward slope), and (2) radiation intensity decreases during the recovery of the E-field after it reaches the maximum (upward slope). The low-frequency envelope of the UHF radiation record results from the high rate of negative leader pulses.

Records of magnetic flux density, $d B / d t$, of rocket-initiated discharges consist of high-rate pulses superimposed on a low- frequency variation (see Fig. 6). The high-rate pulses are associated with current pulses of streamers in the negative stepped leader. They are most pronounced in records immediately following initiation. The low-frequency component in magnetic field changes is associated with continuous current in the expanded channel. This low-frequency variation switches polarity from positive to negative (Fig. 6) when the E-field increasing. Because the magnetic flux density is proportional to dI/dt, the positive polarity corresponds to increasing continuous current ( $\mathrm{dI} / \mathrm{dt}>0$ ) during the period following leader initiation, and the negative polarity corresponds to decreasing current ( $d I / d t<0)$ during the period from the maximum point to channel cutoff. The "fast antenna" record (Fig. 6), which shows changes of current, $I(t)=$ $d Q(t) / d t$, clearly indicates the point of the continuous current cutoff (crossing point of the zero axis), which corresponds to the maximum of the E-field and to the second zero point of the magnetic flux density record.

Examining E-field records of the rocket-initiated discharges under the positive E-field aloft revealed that these discharges do not have a pulse similar to that of the return stroke in the natural positive CG flash. Thus the discharge initiated by the rocket with a trailing and grounded wire is a purely negative leader consisting of continuous current and a stepped (pulse) process.

\section{Discussion}

An ascending rocket connected to the ground with a wire is analogous to an electrode extending into the ambient E-field. This makes a discharge from the tip of the wire monopolar and unidirectional, a sort of "moving electrode." After the connection to the ground is cut off, the lightning channel is equivalent to an isolated conductor placed in the ambient E-field.

The fundamentals of the electrostatic theory of lightning [Kasemir, 1960] are applied here under the simplified assumption of a constant ambient E-field overhead. A conducting lightning channel is connected to the ground as a source of charge and is exposed to an ambient E-field. Thus the charge distribution on the channel is a result of two effects: induced charges caused by the ambient positive field (symmetrical, negative on the top, positive on the bottom), as if the channel is disconnected from the ground, and a uniformly distributed negative charge caused by the connection to the ground (rectangular distribution), as if there is no ambient E-field. The total charge distribution is unipolar and in a shape of triangle with zero at the ground, which has zero potential, and a double value of uniformly distributed charge at the tip (Fig. 7). After the continuous current of the negative leader ceases and the channel is cut off at the ground, channel expansion at the tip via negative leader development continues (evident in radiation records). Cooling of the low, "older" portion of the channel occurs simultaneously. The net effect of leader development on the top and channel cooling on the bottom is still an expansion of the channel. Because of the charge conservation in the isolated conductor, the uniform charge 
deposited when channel was in contact with the ground remains the same during channel expansion. However, with increasing length of the channel, charge per unit length of this uniform charge decreases.

Assume that the induced electric charges, which are distributed linearly, negative on the tip, and positive on the bottom, are growing during channel expansion, and their sum equals zero. Then the total charge on the channel after the cutoff from the ground will be distributed bipolarly (but not symmetrically) with a positive charge first showing up and then growing at the ground end, and still larger negative charge on the tip. The positive charge at the low end of the channel grows in magnitude and moves upward, thus producing the E-field change on the ground that is opposite to that caused by a moving upward negative charge in the beginning of the negative leader. The net effect is seen as a recovery of the E-field. This hypothesis should be tested with simultaneous current measurements.

A bidirectional leader of a positive CG flash before the positive leader touches the ground can be approximated (in a constant vertical E-field) as a conductor with a total zero charge and with induced charges linearly distributed with length and increasing with the channel length (Fig. 8). At the moment of touching the ground (a source of a negative charge), a return stroke provides an additional uniformly distributed negative charge to the channel, so that the total charge distribution has zero charge on the ground level and the maximum negative charge at the tip. In our simplified approach, the maximum negative charge is double that before the touchdown. Thus a return stroke regenerates a negative leader process. After that, the lightning process is the same as when the negative leader is initiated by a rocket with a trailing and grounded wire.

Acknowledgements. The authors appreciate the stimulating discussions of issues presented in this paper with Lothar Ruhnke and Marx Brook.

\section{References}

Kasemir, H.W., Qualitative Uebersicht ueber Potential-, Feld-, und Ladungsverhaltnisse bei einer Plitzentladung in der Gewitterwolke, in Das Gewitter by H. Israel, Acad. Verlags. Ges. Geest and Portig K.-G., Leipzig, German Democratic Republic, 1950.

Kasemir, H.W., A contribution to the electrostatic theory of a lightning discharge, J. Geophys. Res.. 65, 1873-1878, 1960.

Kawasaki, Z.I., T. Kanao, K. Matsuura, M. Nakano, K. Horii, and K.I. Nakamura, The ekectric field changes and UHF radiation caused by the triggered lightning in Japan, Geophys. Res. Lett.. 1711-1714, 1991.

Krider, E.P. and G.J. Radda, Radiation fields wave forms produced by lightning stepped leaders, J. Geophys. Res..80, 2653-2657, 1975.

LeVine, D.M. and R. Moneghini, Effects of channel tortuosity on the electric fields radiated from return strokes, proceedings in Atmospheric Electricity, edit. Ruhnke and Latham, A. Deepak Pub. Co, 345-347, 1983.

Takagi N. and T. Takeuti, Oscillating bipolar electric field changes due to close lightning return strokes, Radio Science, 18, 391-398, 1983. 
Kawasaki and Mazur: Common Physical Processes in Lightning

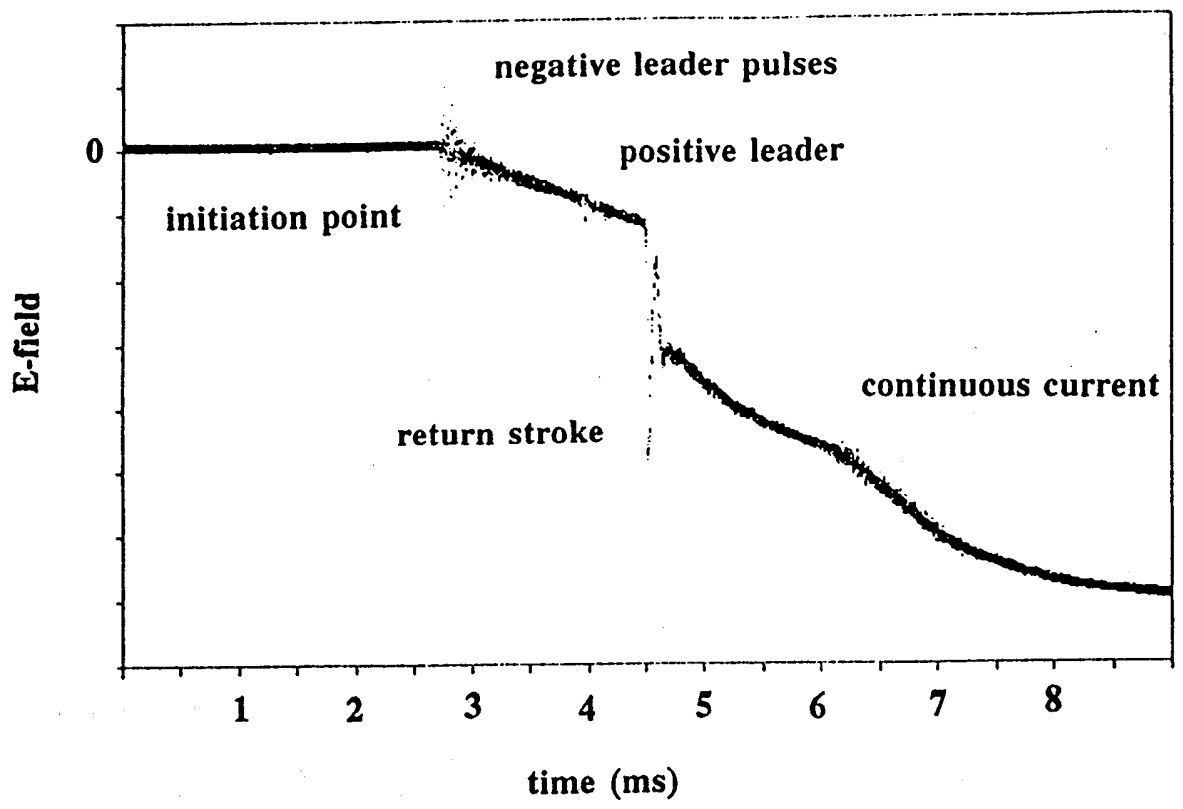

Fig. 1. Electric field changes of a positive CG flash.

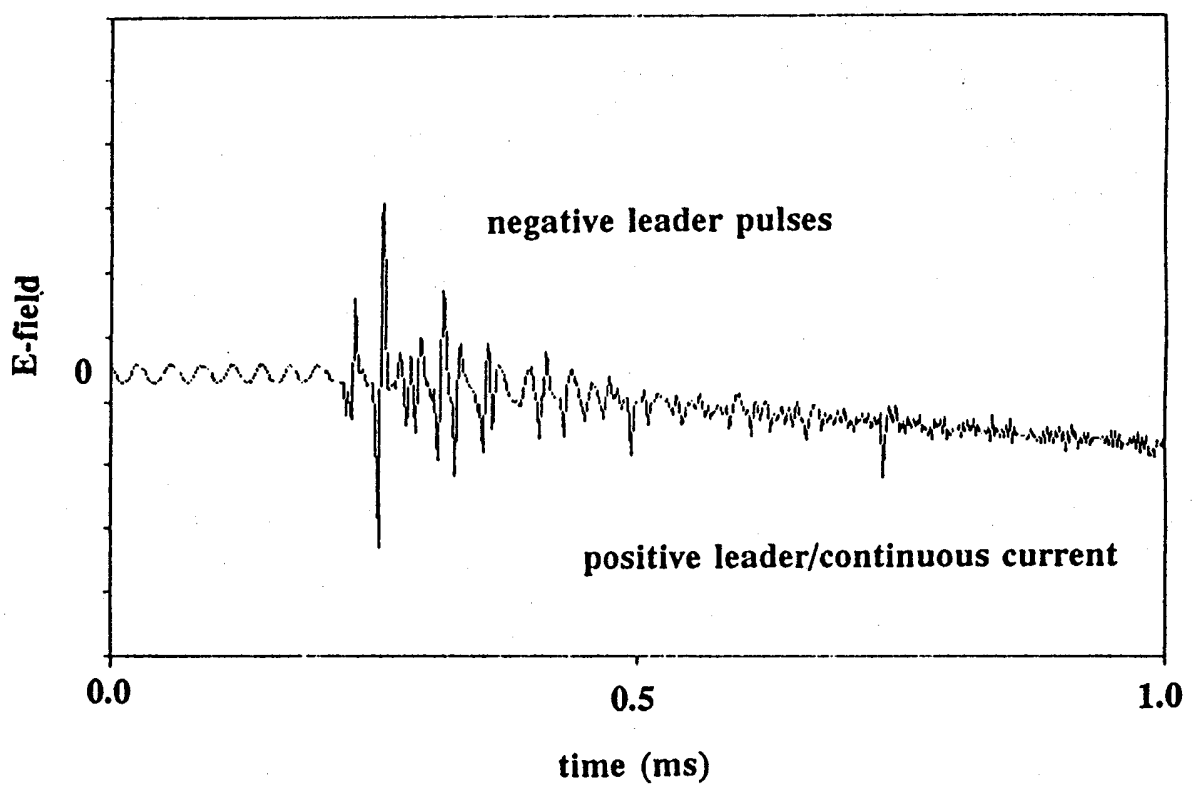

Fig. 2. Initiation of a positive CG flash. 


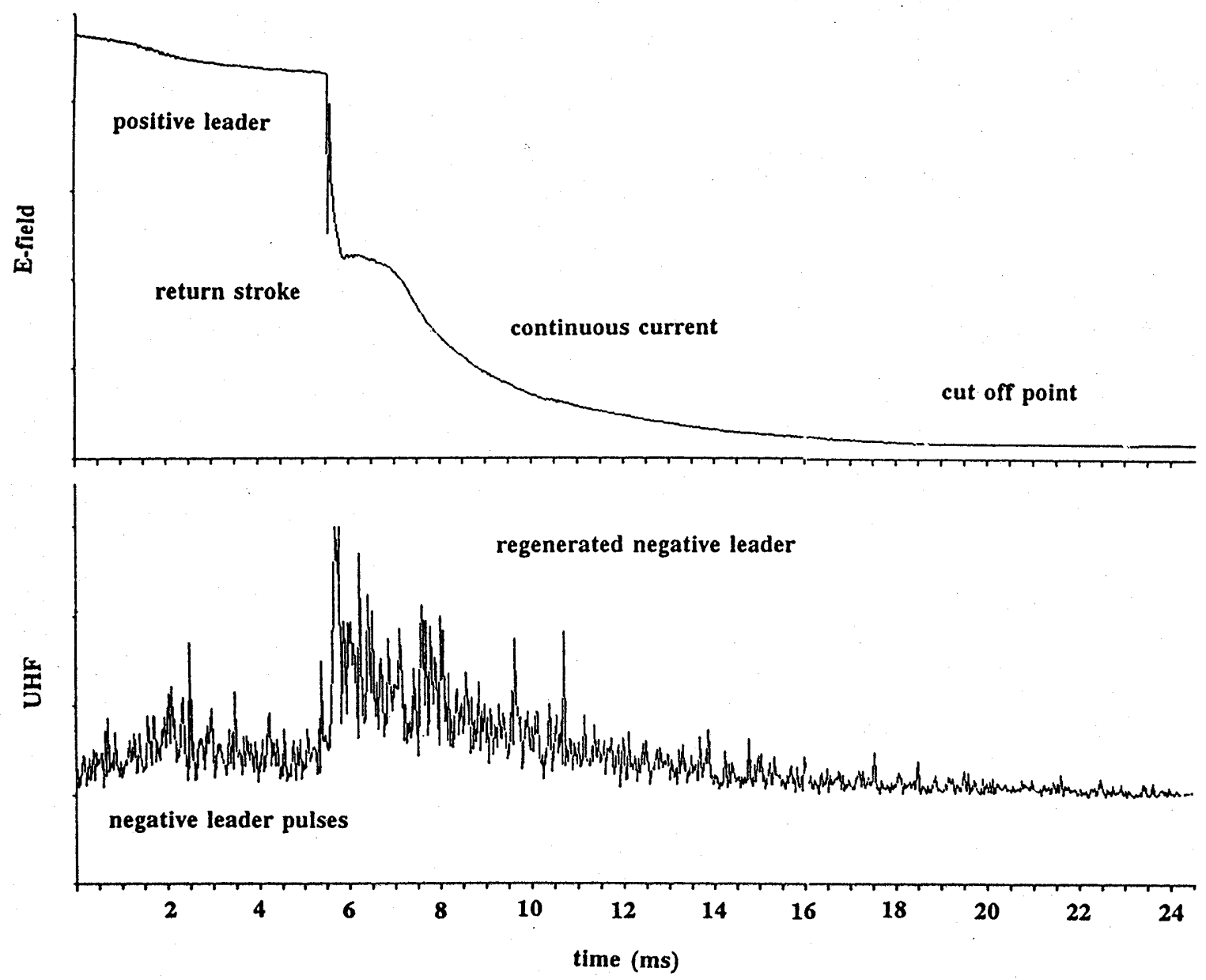

Fig. 3. Electric field changes and UHF radiation of a positive CG flash. 


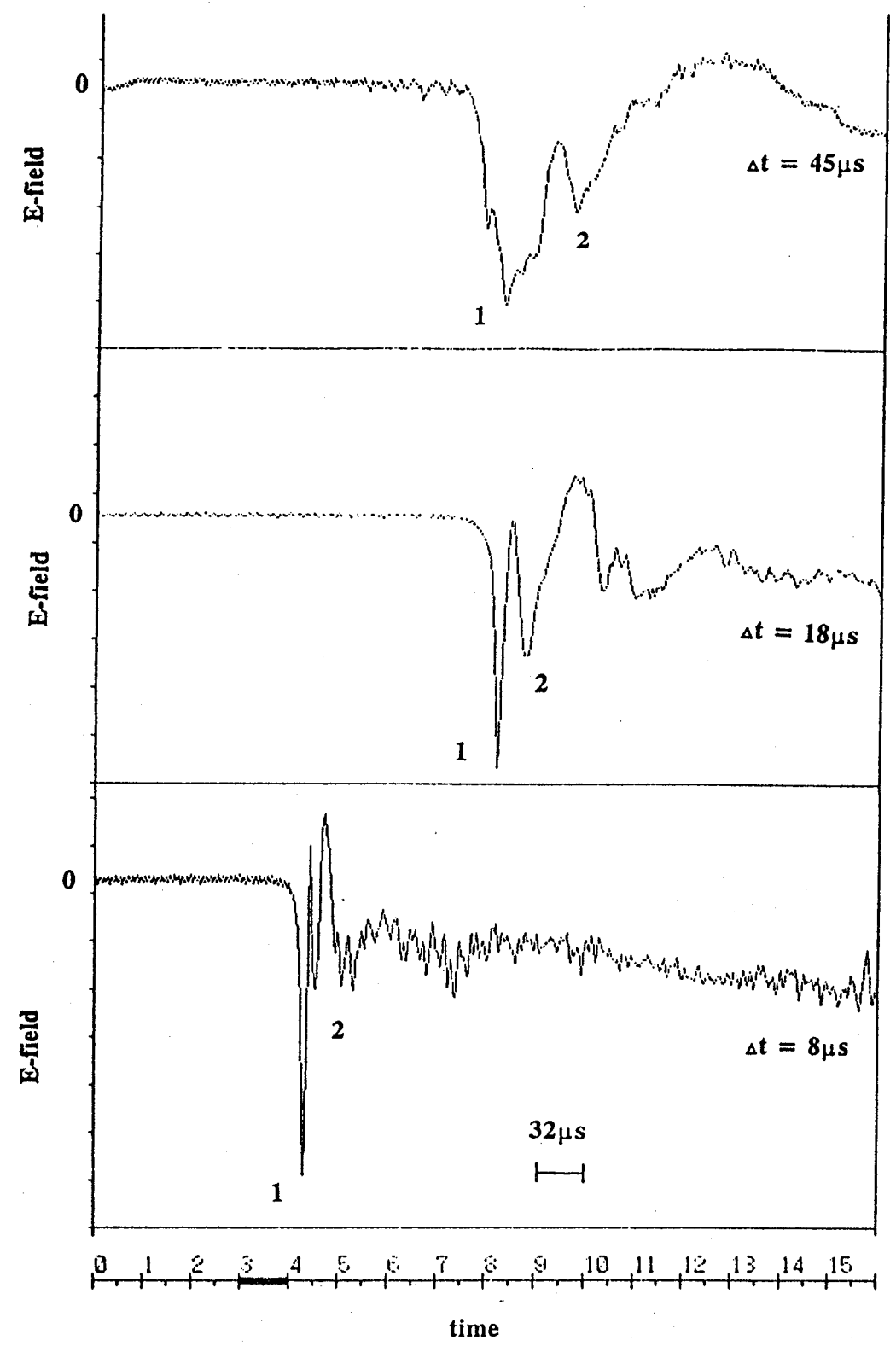

Fig. 4. Electric field waveforms of return strokes in positive CG flashes. Note an oscillating feature in each waveform. Time intervals between peaks (marked 1 and 2) represent period of the oscillation. 

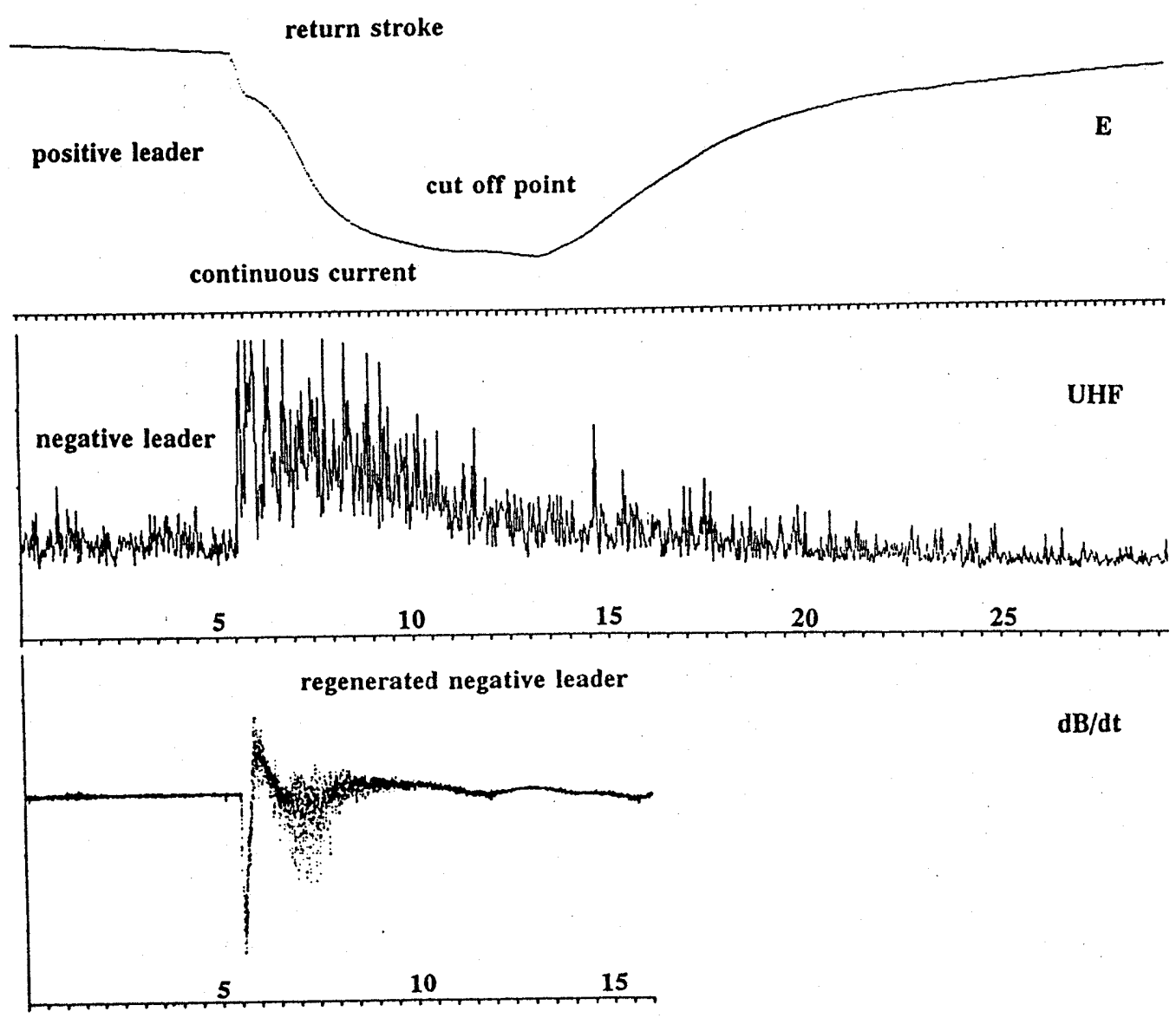

time (ms)

Fig. 5. Positive CG flash: electric field changes (E), UHF radiation (UHF), and magnetic current density $(d B / d t)$. 


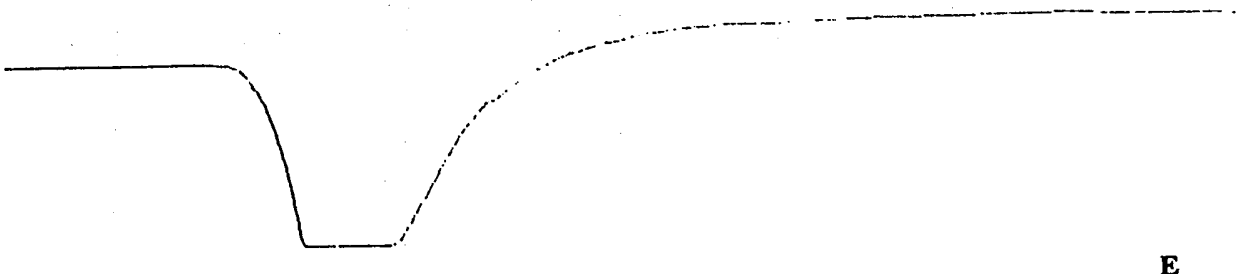

E
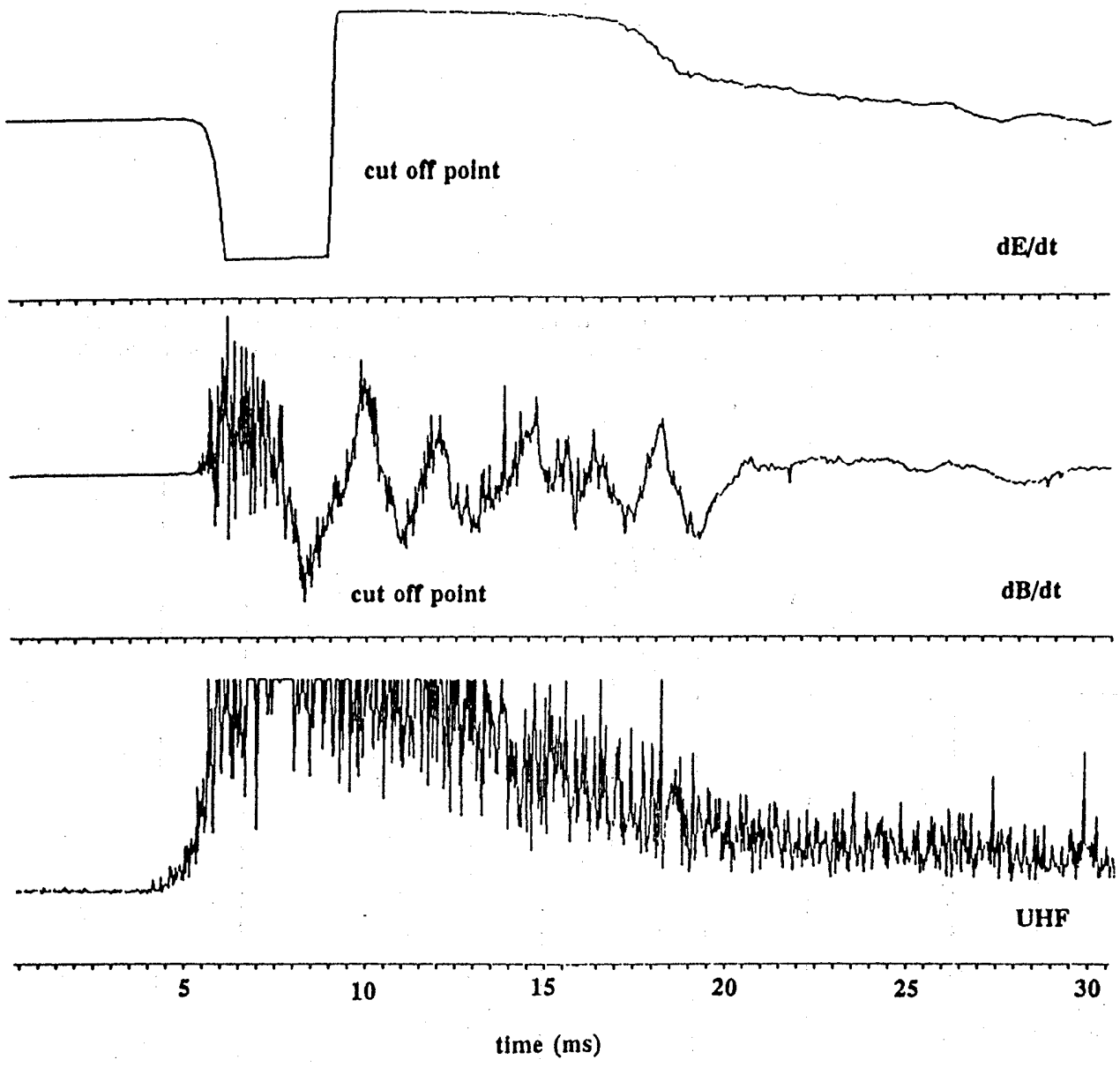

Fig. 6. Negative leader initiated by a rocket with a trailing and grounded wire: electric fleld slow antenna (E), fast antenna (dE/dt) magnetic current density $(d B / d t)$, and UHF radiation (UHF). Although other sets of three sensor records without saturation were available, this record was chosen because it contains all four sensors. 


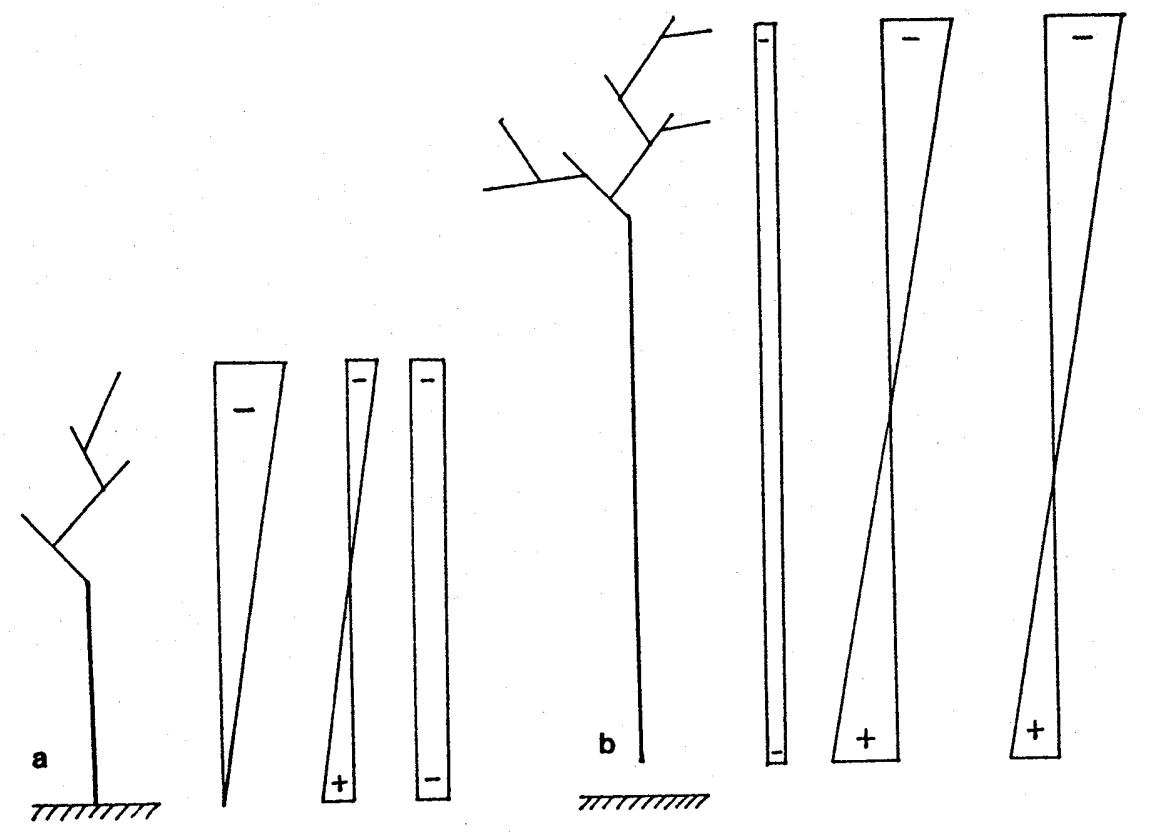

Fig. 7. Negative CG flash, electrostatic theory interpretation. (a) channel connected to the ground, (b) isolated channel after the cease of continuous current.

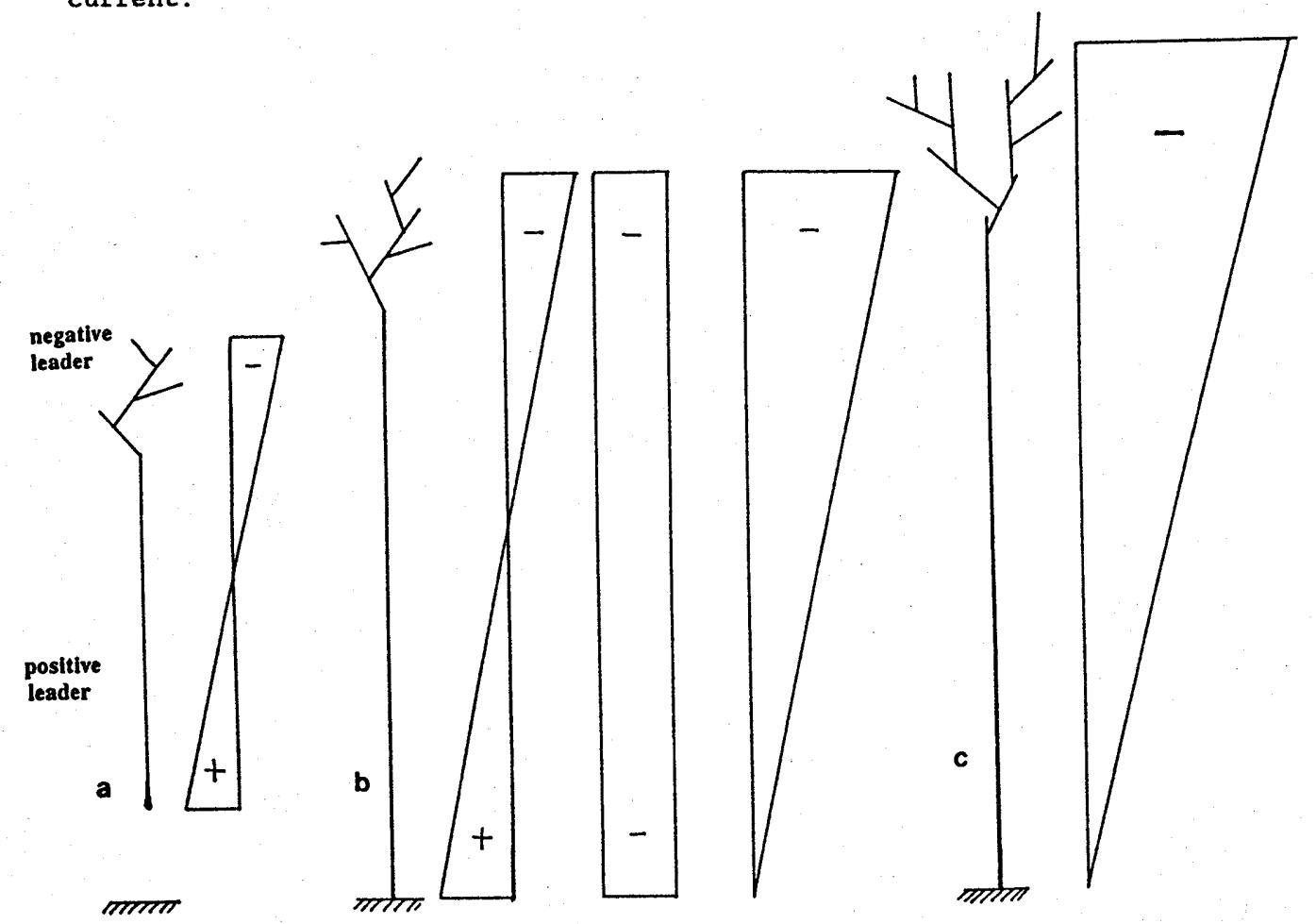

Fig. 8. Positive leader initiated by a rocket with a trailing and grounded wire, electrostatic theory interpretation. (a) bidirectional leader stage, (b) return stroke stage, (c) negative leader stage with channel connected to the ground.

(Received January 30, 1992; Accepted March 1, 1992.) 